\section{Functional observations in vitamin A deficiency: diagnosis and time course of recovery}

VA McBain ${ }^{1,4}$, CA Egan', SJ Pieris², G Supramaniam³, AR Webster ${ }^{1}$, AC Bird ${ }^{1}$ and GE Holder ${ }^{1}$

\begin{abstract}
Aims To describe the effects of vitamin A deficiency (VAD) on retinal function and the subsequent recovery following treatment in three patients with systemic conditions (two with Crohn disease; one secondary to IgE syndrome).
\end{abstract}

Methods Electrophysiological testing (including pattern electroretinogram, PERG; electroretinogram, ERG; visual-evoked potential) established the diagnosis of VAD. Repeat testing was carried out in two patients to monitor the time course of recovery following intramuscular vitamin A injection. The third patient had repeat recordings following 13 months of oral supplementation. Results All three patients initially displayed a characteristic absence of rod function associated with VAD. In addition, delayed and reduced amplitude cone ERGs, loss of short wavelength cone (S-cone) function and subnormal macular function were observed in two patients. Restoration of rod and generalised cone function was rapid in the two patients who received intramuscular injection, with normalisation of some electrophysiological responses after only 3 days. Normal S-cone amplitudes and cone latencies were reached within 12 days of vitamin A injection. Macular function returned to within normal limits by 12 days postinjection in one patient, but remained mildly subnormal in the second patient. Full recovery was present after 13 months oral supplementation in the third patient.

Conclusions Novel observations regarding dark-adapted cone function, S-cone function, and PERG are presented. The differences between the effects of VAD on rod and cone function, and their rate of recovery, may reflect differences in the visual cycle between the two photoreceptor classes. The importance of rapidly and accurately diagnosing VAD, a treatable condition, is noted.

Eye (2007) 21, 367-376. doi:10.1038/sj.eye.6702212; published online 9 December 2005

Keywords: vitamin A; electroretinogram; rod function; pattern ERG; retina

\section{Introduction}

Vitamin A is a fat-soluble vitamin essential for the formation of photoreceptor visual pigment (rhodopsin). During the early stages of vitamin A deficiency (VAD), stores of vitamin A in the liver and blood are depleted before

photoreceptor outer segments become deficient in functional rhodopsin. With prolonged

deficiency, there is loss of visual sensitivity and eventual shrinkage and loss of photoreceptor outer segments. ${ }^{1-4}$ Night blindness is usually the first clinical presentation of VAD, which may result from poor dietary intake, decreased intestinal absorption (eg following surgical intervention for Crohn disease or morbid obesity), defective transportation, or liver disease. ${ }^{5-9}$ The fundi of chronic VAD patients may display multiple white or grey-white spots scattered in the peripheral retina, which resolve with supplementation. ${ }^{10}$ However, the majority of published VAD cases have normal ocular examinations. ${ }^{5,8}$ The diagnostic tests that may reveal VAD are the electroretinogram (ERG) and dark adaptometry. Dark adaptometry demonstrates elevated rod and cone thresholds, with rods more severely affected. ${ }^{4-9}$

Electrophysiological tests have been reported to show reduced or undetectable rod ERGs with reduced amplitude cone ERGs of normal implicit time..$^{5-9,11,12}$ Fortunately, night blindness secondary to VAD is a reversible retinal condition and a full recovery is reported in
${ }^{1}$ Moorfields Eye Hospital London, UK

${ }^{2}$ Bedford Hospital, Bedford, UK

${ }^{3}$ Watford General Hospital, Watford, UK

Correspondence: Dr GE Holder, Department of Electrophysiology, Moorfields Eye Hospital, 162, City Road, London, EC1V 2PD, UK

Tel: + 44207566 2120;

Fax: +44 207566 2556;

E-mail: Graham.holder@

moorfields.nhs.uk

${ }^{4}$ Current address:

Department of

Ophthalmology, Institute of Medical Sciences, University of Aberdeen, Foresterhill, Aberdeen, UK

Received: 25 May 2005 Accepted in revised form: 31 October 2005;

Published online:

9 December 2005 
nearly all patients following parenteral or oral vitamin A supplementation. Accurate and early diagnosis is therefore of great clinical relevance.

We report the findings in three patients with VAD. In two adult cases with malabsorption, the rapid recovery of retinal function following vitamin A injection was documented with detailed electrophysiological recordings. Novel observations on the effects of VAD on pattern electroretinogram (PERG), S-cone ERGs, cone response implicit times, and colour contrast sensitivity (CCS) are described. The third case, a child with severely restricted diet consequent upon IgE syndrome, showed full recovery after 13 months oral supplementation.

\section{Methods and case reports}

Electrophysiological studies were performed prior to treatment in all three patients as part of the diagnostic process. In cases 1 and 2, recordings were repeated soon after intramuscular vitamin A injection (days 3 and 12), but in case 3, a child who received oral supplementation, recordings were repeated at 13 months. The study was conducted according to the Tenets of the Declaration of Helsinki.

\section{Case 1}

A 59-year-old man presented to another hospital with night blindness of 2 months duration and was referred for electrophysiological testing. Initially, he was aware of bilateral ring-shaped scotomata that progressed within weeks to total night blindness with the temporal field last affected. He began to experience some difficulties with his daytime vision prior to presentation. Previous medical history included several bowel resections for Crohn disease. The patient was taking an oral ionbinding resin (Questran ${ }^{\mathrm{R}}$ ), but oral vitamin supplementation had been ceased 3 months prior to presentation.

Visual acuity was $6 / 6$ on the right and $6 / 9+1$ on the left with spectacles. Ophthalmic examination was unremarkable; ERGs suggested VAD, confirmed by haematological testing (serum vitamin A was $0.1 \mu \mathrm{mol} / \mathrm{l}$ (normal 1.5-2.5)). In addition, vitamin E was $12.3 \mu \mathrm{mol} / 1$ (normal 12.0-28.0), and 25-hydroxy-vitamin D was $72 \mathrm{nmol} / 1$ (normal 40-195). Intramuscular vitamin A supplementation was commenced with 100000 international units and followed up with two further doses at weekly intervals. The patient described a dramatic improvement in symptoms following vitamin A injection, in particular his night vision improved within 2 days. Vision improved to $6 / 6$ in both eyes with spectacles. The serum vitamin A level 2 weeks after the final injection had improved to $2.6 \mu \mathrm{mol} / 1$ (normal
1.5-2.5). Vitamin E and D levels, which previously fell within the normal range, were not remeasured. The patient was subsequently maintained on oral vitamin A supplementation.

\section{Case 2}

A 55-year-old man was referred with vague visual symptoms, principally seeing 'white as green'. Direct questioning elicited poor vision in dim illumination over the previous months. He had previously been investigated for suspected optic nerve dysfunction, which included an MRI scan, but no abnormality had been detected. His visual symptoms included bilateral central field defects, most noticeable on waking in the morning, which 'dissolved' on entering a brightly lit room. The patient had also noticed slight photophobia and 'flickering vision'. There was a history of anterior uveitis 5 years prior to presentation. Questioning revealed a 20-year history of Crohn disease and multiple bowel resections. Malabsorption had previously manifested as water and electrolyte imbalance. The patient was taking a calcium supplement $\left(\mathrm{Calcichew}^{\mathrm{R}}\right)$, antimotility drug (Loperamide hydrochloride), antacid (magnesium carbonate), antiproliferative immunosuppressant (Azathioprine), and iron supplement, but the only vitamin supplementation was vitamin D (Calcitriol).

On examination, visual acuity in the amblyopic right eye was $6 / 36$, improving to $6 / 18$ with pinhole and hypermetropic correction (plus 2D). The uncorrected visual acuity in the left eye was $6 / 6$. No fundus abnormalities were identified. After ERGs suggested $\mathrm{VAD}$, serum vitamin $\mathrm{A}$ was recorded at $0.19 \mu \mathrm{mol} / \mathrm{l}$ (normal 1.05-2.80). In addition, vitamin E was $5.7 \mu \mathrm{mol} / 1$ (normal 11.5-35) and vitamin $\mathrm{K}$ was undetectable (normal 0.15-1.55 $\mu \mathrm{mol} / \mathrm{l}$ ). Treatment was commenced as for case 1. Following treatment, the patient reported a dramatic improvement in all his symptoms starting within $12 \mathrm{~h}$ of the first vitamin A injection. Figure 1 shows the sketches spontaneously drawn by the patient to illustrate the dramatic subjective improvement in central visual function following the first injection (LE only). The serum vitamin A level at 4 months was $2.59 \mu \mathrm{mol} / 1$ (normal 1.05-2.80 $\mu \mathrm{mol} / \mathrm{l}$ ) and vitamin $\mathrm{K}$ was $0.11 \mu \mathrm{mol} / 1$ (normal $0.15-1.55 \mu \mathrm{mol} / \mathrm{l}$ ). Vitamin E was not remeasured.

\section{Case 3}

A 9-year-old boy was referred for electrophysiology testing to investigate possible problems related to malnutrition from early infancy. He had experienced several months of difficulty with night vision. The 


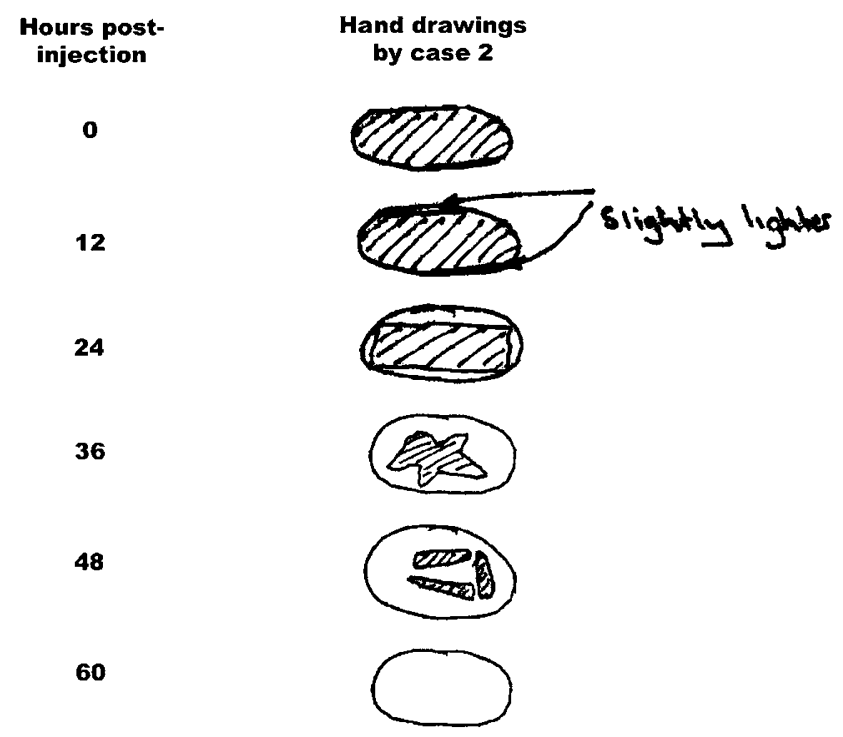

Figure 1 Hand drawings by case 2 (LE) illustrating the subjective recovery of his central visual field defect following supplementation. The patient began to notice a slight improvement at the very edges of his visual field only $12 \mathrm{~h}$ after the first vitamin A injection.

patient suffered IgE syndrome with multiple severe food allergies. His diet was restricted to rice, potatoes, sweet corn, cabbage, yam, lemon and lime jam and juice, cucumber, lettuce, white fish, salmon, sunflower and olive oil, apple, pear, and melon. His medications comprised those for asthma (Seretide ${ }^{\mathrm{R}}$, Ventolin ${ }^{\mathrm{R}}$ ), the topical emollients and corticosteroid treatments (Propaderm $^{\mathrm{R}}$, Aveeno ${ }^{\mathrm{R}}$ oil, Polytar ${ }^{\mathrm{R}}$ shampoo, Diprobase $^{\mathrm{R}}$ ), and an antihistamine (Piriton ${ }^{\mathrm{R}}$ ). On examination, visual acuities were $6 / 5$ right; $6 / 6$ left.

Fundus examination was normal. Electrophysiological testing suggested VAD, and serum vitamin A levels were $0.1 \mu \mathrm{mol} / 1$ (normal $0.9-1.7 \mu \mathrm{mol} / 1$ ). Other vitamin levels were not available. Owing to his age and the possibility of an allergic reaction, he was treated with oral vitamin $\mathrm{A}$ supplementation (Ketovite ${ }^{\mathrm{R}}$ liquid) rather than intramuscular injection. After 3 months of treatment, the patient was followed up by the referring consultant and found to be feeling much better and to have no night vision problems. The patient continued to report his night vision as 'back to normal' at the 13-month electrophysiology follow-up visit. Serum vitamin A level after 3 months treatment was $1.04 \mu \mathrm{mol} / 1$ (normal $0.9-1.7 \mu \mathrm{mol} / 1)$.

\section{Electrophysiology}

In all three cases, extended full-field ERGs and PERGs were performed to incorporate the ISCEV Standard recommendations. ${ }^{13,14}$ Gold foil corneal recording electrodes were used with ipsilateral outer canthus reference electrodes and a forehead ground. Full-field ERGs were carried out following maximal dilation (topical tropicamide $1 \%$ and phenylephrine $2.5 \%$, or tropicamide alone if phenylephrine was contraindicated). Further analysis of the photopic pathways was achieved using long duration stimuli to examine separately the photopic ON and OFF systems, ${ }^{15}$ and utilised a $200 \mathrm{~ms}$ amber stimulus $\left(650 \mathrm{~cd} / \mathrm{m}^{2}\right)$ with a green background $\left(160 \mathrm{~cd} / \mathrm{m}^{2}\right)$ at $2.5 \mathrm{~Hz}$. The S-cone system was examined using a $5 \mathrm{~ms}$ blue stimulus $\left(80 \mathrm{~cd} / \mathrm{m}^{2}\right)$ with an amber background $\left(650 \mathrm{~cd} / \mathrm{m}^{2}\right)$ at $1 \mathrm{~Hz} .{ }^{16}$ PERG was performed undilated. A black and white reversing checkerboard stimulus (rate $4.5 / \mathrm{s}$, check size $45^{\prime}$, field size $15^{\circ} \times 15^{\circ}$ ) was used. Michelson contrast was $98 \%$, and mean luminance was $80 \mathrm{~cd} / \mathrm{m}^{2}$. In addition visual-evoked potentials (VEPs), and CCS were examined in case 2. Pattern VEPs (PVEPs) used a black and white reversing checkerboard stimulus (rate 2/s, check size $50^{\prime}$, field size $15^{\circ} \times 15^{\circ}$ ). Michelson contrast and mean luminance were as above. CCS was measured using a custom-built system ${ }^{17}$ to examine the colour contrast thresholds in each of the main three colour confusion axes (protan, deutan, and tritan).

\section{Results}

\section{Pretreatment}

The electrophysiological findings prior to treatment are shown in Figures 2-4. In all three cases, rod-specific responses were bilaterally undetectable (scotopic rod) 

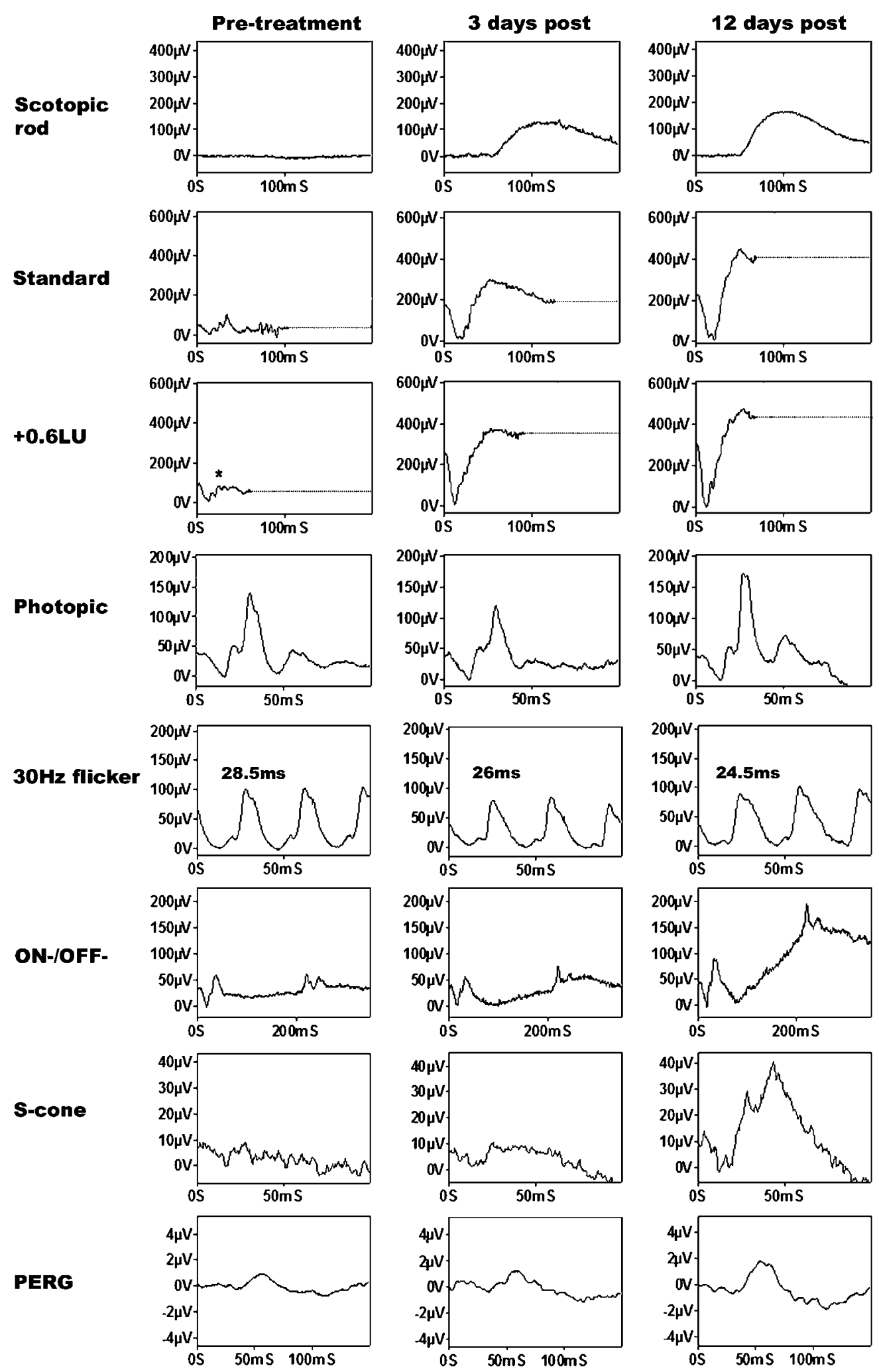

Figure 2 Pre- and post-treatment (days 3 and 12) electrophysiology for case 1. Pretreatment: Scotopic rod responses are undetectable. Standard maximal mixed rod-cone response is grossly subnormal, and resembles the photopic cone response waveform. The asterisk highlights the additional reduction in b-wave amplitude with increasing light intensity. Photopic ERGs are of borderline amplitude and increased latency. Long duration ON and OFF ERGs are similarly affected. S-cone ERGs are undetectable. PERG is subnormal. Post-treatment: Scotopic rod, standard responses, and generalised cone ERGs recovered within 3 days of vitamin A supplementation. S-cone and macular function normalised by day 12. 


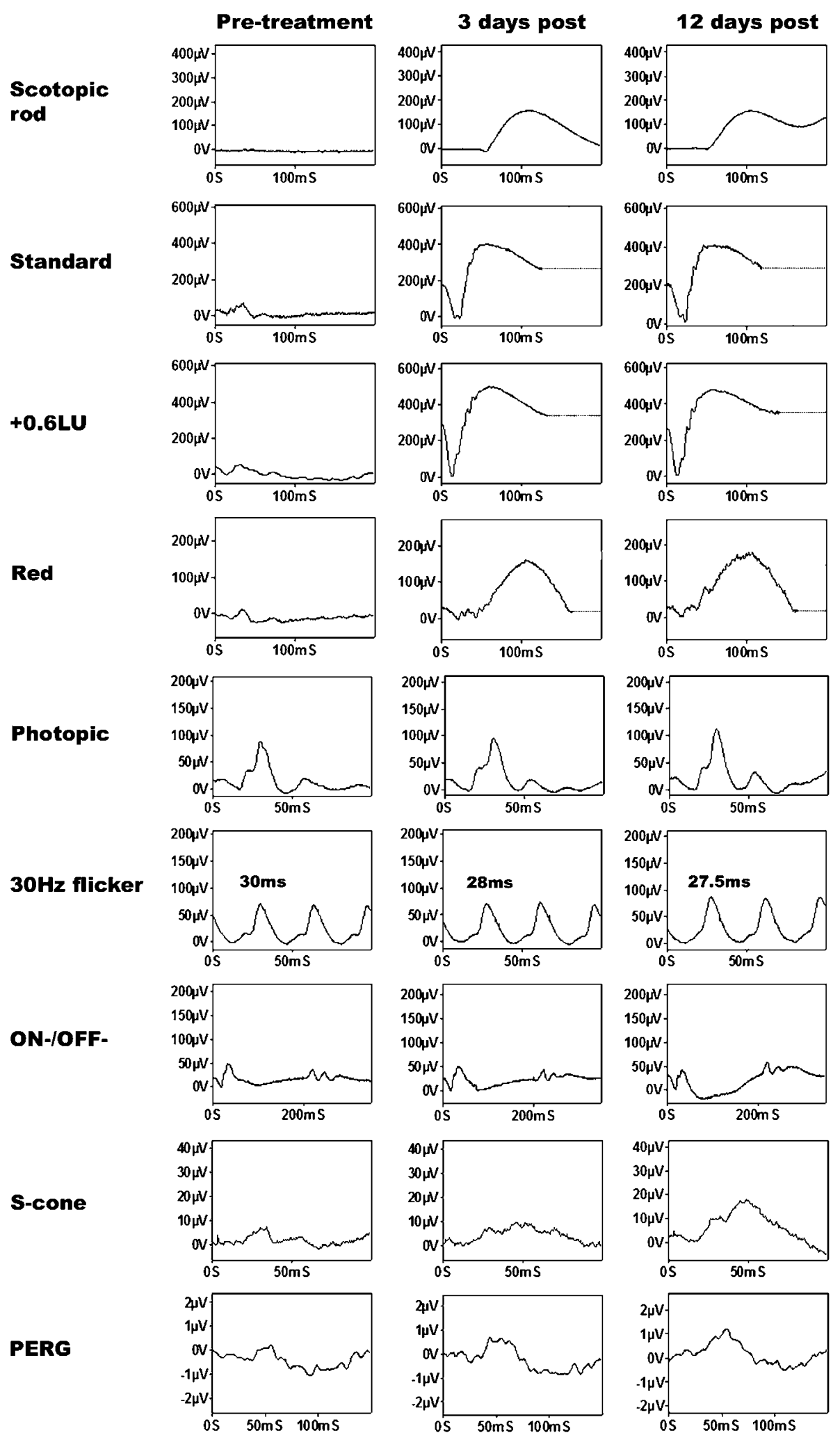

Figure 3 Pre- and post-treatment (days 3 and 12) electrophysiology for case 2. Pretreatment: Scotopic rod responses are undetectable. Standard mixed rod-cone response is grossly subnormal and resembles the photopic cone response waveform. Stimulation with a red light under dark-adapted conditions also revealed only cone-derived activity, but no definitely detectable rod-derived component. Photopic ERGs are of borderline amplitude and increased latency. Long duration ON and OFF ERGs are similarly affected. S-cone ERGs are undetectable. PERG is subnormal. Post-treatment: Scotopic rod, standard responses, and generalised cone ERGs recovered within 3 days of vitamin A supplementation. S-cone function normalised by day 12. PERG remained mildly subnormal at day 12 for the patients' age. 


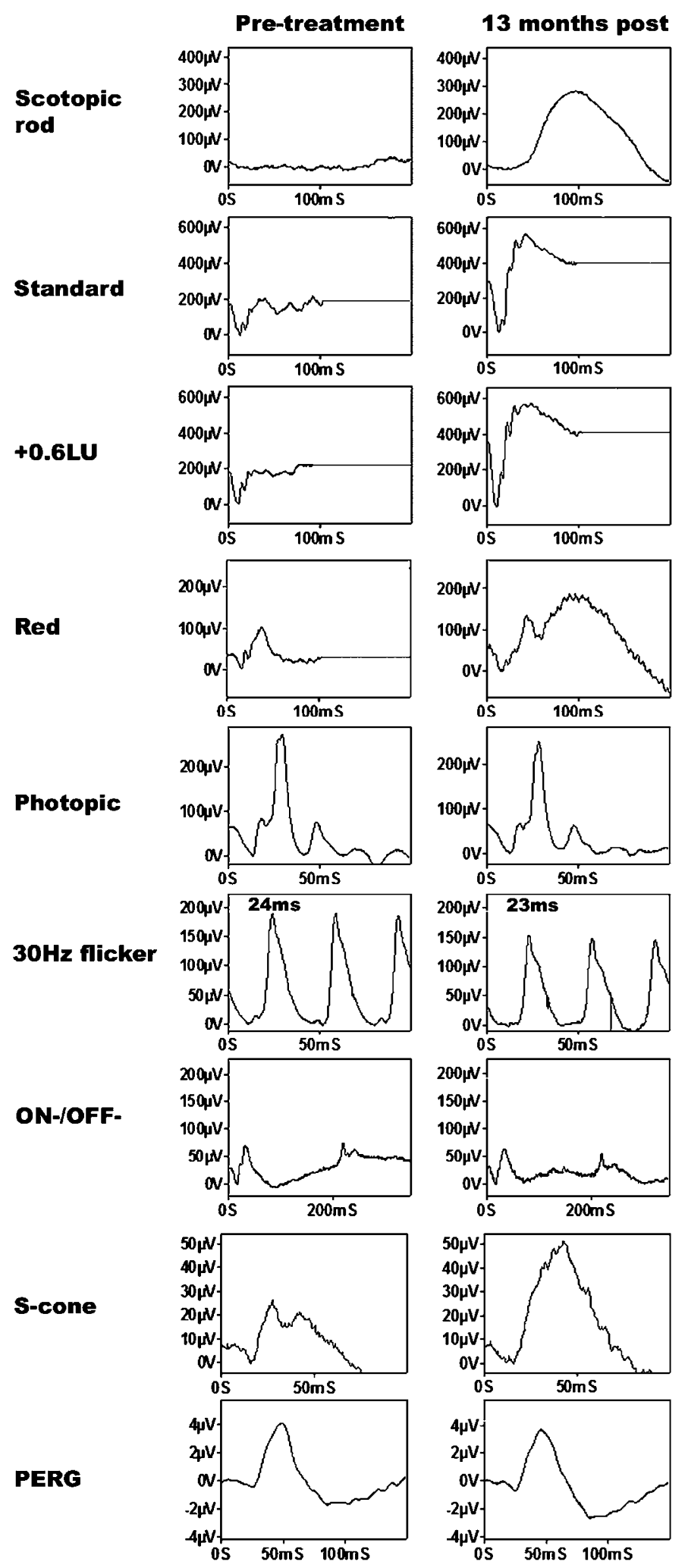

Figure 4 Pre- and post-treatment (13 months) electrophysiology for case 3. Pretreatment: Scotopic rod responses are undetectable. Standard mixed rod-cone response is grossly subnormal, and resembles the photopic cone response waveform. Stimulation with a red light under dark-adapted conditions also reveals only cone-derived activity with no definitely detectable rod-derived component. Photopic ERGs (flicker, transient, and ON/OFF) and PERG are normal. S-cone ERGs are borderline. Post-treatment: Scotopic rod and standard responses normalised following 13 months of oral supplementation. S-cone amplitudes doubled. Generalised cone and macular function were unchanged from pretreatment recordings. 
and the ERGs at brighter intensities (standard) resembled those derived solely from the cone system, displaying a short latency a-wave with rapid fall-off following the peak of a short latency b-wave (Figures 2-4). Additional increment of the stimulus intensity $(+0.6 \mathrm{LU})$ resulted in further reduction of the $b$-wave amplitude relative to an increasing a-wave (asterisk, Figure 2, column 1). Stimulation with a red light under dark-adapted conditions revealed only a clear cone-derived component with no definitely detectable rod-derived component (Figures 3 and 4). Cone ERGs in cases 1 and 2 (photopic, flicker) were of increased latency and borderline/ subnormal amplitude, but considerably better preserved than rod function; S-cone function was undetectable and ON-OFF ERGs borderline/subnormal (Figures 2 and 3). All cone ERGs (photopic, flicker, ON-OFF, and S-cone) were normal in case 3 (Figure 4). PERG P50 and N95 component amplitudes were bilaterally subnormal in cases 1 and 2 (Figures 2 and 3), but normal in case 3 (Figure 4).

CCS testing in case 2 revealed extremely elevated thresholds along all three axes in both eyes (Figure 5a). Pattern VEPs (not shown) were also delayed, almost certainly consequent upon the reduced PERG. Flash VEPs were unremarkable (not shown).

\section{Post-treatment}

Cases 1 and 2 were examined during the acute phase of recovery following the first vitamin A injection. Case 3 was examined following 13 months oral vitamin A supplementation.

\section{Case 1}

The data are shown in Figure 2. All ISCEV standard fullfield ERGs had recovered to just within normal limits at 3 days post-treatment and fell well within the normal range at 12 days (Figure 2). There was a clear rod-specific $\mathrm{b}$-wave and the standard responses displayed the typical mixed rod/cone waveform. Cone ERGs were of normal amplitude and implicit time. S-cone ERGs remained subnormal at 3 days post-treatment, but fell within normal limits at 12 days post-treatment (Figure 2). PERG P50 and N95 component amplitudes remained subnormal at 3 days post-treatment, but normalised by day 12.

\section{Case 2}

The data are shown in Figure 3. All ISCEV standard fullfield ERGs had completely normalised by 3 days posttreatment and were unchanged at day 12. S-cone function was just detectable at 3 days post-treatment, but was within normal limits at 12 days post-treatment. PERG P50 and N95 component amplitudes were significantly improved at 3 and 12 days (Figure 3), and had further improved 3 months post-treatment, but remained mildly subnormal at 6 months post-treatment (Figure 5b). CCS thresholds significantly improved in all three axes 3 days post-treatment, but remained mildly elevated (Figure 5a). At 12 days post-treatment, CCS thresholds were only borderline subnormal, and by 3 months post-treatment, had normalised (Figure 5a). Pattern VEP latency remained bilaterally delayed (not shown), in keeping with the subnormal PERG P50.

\section{Case 3}

The data are shown in Figure 4. All ISCEV standard fullfield ERGs had recovered to well within normal limits at 13 months postsupplementation (see Figure 4). There was a clear rod-specific b-wave and the standard responses displayed the typical mixed rod/cone waveform. Cone ERGs were normal and unchanged from the pretreatment recordings. S-cone ERGs, although within normal amplitude limits pretreatment, significantly increased following 13 months of supplementation. PERG P50 and N95 component amplitudes were also normal and unchanged from the pretreatment recordings.

\section{Discussion}

Three cases of VAD were ascertained electrophysiologically. Two patients were re-examined during the acute phase of recovery following intramuscular vitamin A injection; the third, for whom intramuscular vitamin A was not clinically felt to be appropriate, was followed up at 13 months following oral vitamin A supplementation. Novel observations in relation to PERG, generalised cone ERGs, S-cone ERGs, and colour vision are presented.

All three patients displayed characteristic ERG changes prior to treatment. Rod function was severely reduced or undetectable in keeping with previous studies. ${ }^{5,6,9,11,12}$ The delayed cone ERG latency, initially present in two patients but normalising within 3 days of vitamin A injection, has not been described previously. In the third case, the cone ERGs were normal. The effects of VAD on S-cone function also appear to be undescribed previously. S-cone function, like rod function, was undetectable in the VAD state of cases 1 and 2. The visual disturbance ('seeing white as green') described by case 2 may be related to this selective loss of S-cone function. Recovery of S-cone function was more prolonged than generalised cone system recovery (12 days posttreatment vs 3 days post-treatment), although, interestingly, the patient (case 2) reported that the above visual symptom ('seeing white as green') was no longer present 3 days post-treatment. The S-cone ERG 

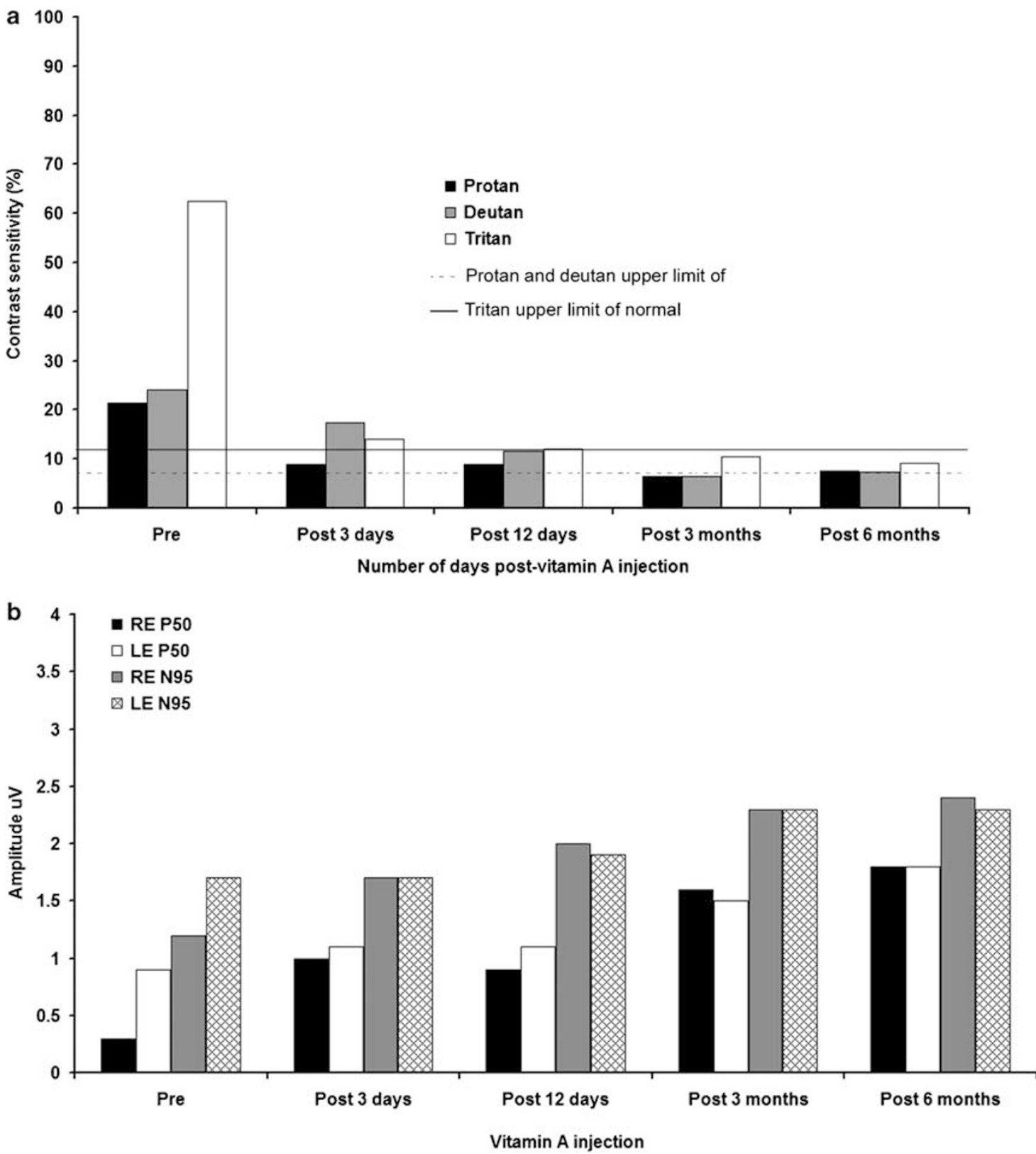

Figure 5 Graphs illustrating CCS thresholds and PERG in case 2 pre- and post-treatment (3 days, 12 days, 3 months, and 6 months). (a) CCS thresholds for protan, deutan, and tritan. All three axes are grossly elevated pretreatment in both eyes (LE illustrated). At day 1,2 post-vitamin A treatment all three colour axes are borderline, but normalise after 3 months. (b) PERG P50 and N95 component amplitudes. A concordant P50 and N95 component amplitude increase is seen with each visit, but absolute values remain borderline subnormal at 6 months for the patients' age.

amplitude in the third patient, although within normal limits pretreatment, significantly increased following oral supplementation. The rapid recovery of generalised cone function (day 3 of treatment), however, parallels the time course of rod system recovery and the reported dramatic recovery symptomatically by both cases 1 and 2 .

Recovery of loss of central cone function was, however, more prolonged. Macular function only normalised by day 12 in case 1, and remained mildly subnormal even at 6 months post-treatment in case 2 (Figure 5b), although the function appeared to still be improving.

These changes correlate with the time course of the subjective patient information and tests. For example, the recovery of Snellen acuity (cases 1 and 2), the rapid resolution of subjective central field defects (case 2, Figure 1), and the improvement of CCS by day 12 
post-treatment. It is possible that the period or severity of prolonged VAD in case 2 caused more profound effects on macular cone function, possibly irreparable damage. ${ }^{2,6,11}$ A coexistent maculopathy cannot absolutely be excluded, but there was no clinical evidence to suggest this and fundus examination was unremarkable. The third patient had normal PERGs paralleling the normal full-field cone responses. The differences in the duration and cause of VAD, that is, surgically induced malabsorption in two patients and malnutrition in the third, may account for less severe effects in the dietaryrestricted patient such that ISCEV standard cone ERGs remained normal.

In all three patients, the pretreatment retinae behaved in a functionally isolated cone state. This provided the opportunity to study dark-adapted cones in the absence of modifying factors from rod function. All brighter white flash ERGs had a waveform similar to that of the cone response to red light under dark adaptation, and in keeping with a cone system origin. The relative changes in a- and b-wave amplitude with increasing stimulus intensity resemble those described under photopic conditions as the 'photopic hill'. ${ }^{18,19}$ This phenomenon thus appears to be a property of cones rather than be related to the adaptive state of the retina. Perlman et $a l^{5}$ and Carr ${ }^{10}$ previously reported a similarity between the scotopic and photopic responses. Although Perlman et $a l^{5}$ suggested that the 'negative' ERG could indicate that signal transmission in the inner retina was affected, it is likely that their data merely reflect the 'photopic hill' phenomenon in a dark-adapted functionally coneisolated retina. A similar mechanism may explain the 'negative' ERG seen in some cases of fundus albipunctatus, where the retina after ISCEV Standard dark adaptation also remains in a functionally coneisolated state due to the slow regeneration of rhodopsin consequent upon RDH5 mutation. ${ }^{20}$

The recovery of retinal function following treatment is rapid. Cases 1 and 2 regained almost normal ERG rod function within 3 days of vitamin A injection with complete recovery within 12 days. The PERG remained subnormal in case 2 . This is not well documented previously. One study describes normalisation of ERGs within 3 days of initiation of vitamin A therapy, but only report a single ERG response. ${ }^{6}$ Other authors report slower recovery. ${ }^{5,11,12}$ In the animal model, Katz et $a l^{1}$ report rhodopsin levels recovering to $30 \%$ of normal after just 1 day of supplementation (ERGs showed commensurate increase) in retinoid-deprived rats, which is at rates far greater than the normal rate of new opsin synthesis. The findings in our patients mimic the animal model of Katz et al ${ }^{1}$ with respect to recovery of rod function. The previously described order of recovery of photoreceptor function following vitamin A supplementation has been cone function followed by peripheral rods and then perifoveal rods. ${ }^{4,9}$ However, in our experience, the order of recovery is better described as rod and generalised cone function followed by macular and S-cone function. Although the cone dysfunction in two of our patients was more widespread than previously reported, there was a much more profound effect on rod function. Cone visual pigments are more rapidly synthesised than the rod pigments, and thus, with decreasing supplies, cones may capture vitamin $\mathrm{A}$ at the expense of rods. Alternatively, cones might be able to access stores of vitamin A denied to rods, for example, Mata et $a l^{21}$ recently demonstrated a novel pathway for opsin photopigment regeneration involving cone and Müller cells, which may explain the relative resistance of cones in VAD.

This study reports novel observations in relation to previously unrecognised aspects of retinal dysfunction in VAD. In particular, the behaviour of what appears to be functionally isolated cones in the absence of rod function and the effect of VAD on S-cone and macular function. VAD should be suspected in any patient with visual problems in whom there is a history of any surgery likely to result in malabsorption of fat-soluble vitamins, or in nutritionally challenged patients.

\section{References}

1 Katz ML, Chen DM, Stientjes HJ, Stark WS. Photoreceptor recovery in retinoid deprived rats after vitamin A replenishment. Exp Eye Res 1993; 56: 671-682.

2 Carter-Dawson L, Kuwabara T, O'Brien PJ, Bieri JG. Structural and biochemical changes in vitamin A-deficient rat retinas. Invest Ophthalmol Vis Sci 1979; 18: 437-446.

3 Noell WK, Delmelle MC, Albrecht R. Vitamin A deficiency effect on retina: dependence on light. Science 1971; 172: 72-75.

4 Kemp CM, Jacobson SG, Faulkner DJ, Walt RW. Visual function and rhodopsin levels in humans with vitamin A deficiency. Exp Eye Res 1988; 46: 185-197.

5 Perlman I, Barzilai D, Haim T, Schramk A. Night vision in a case of vitamin A deficiency due to malabsorption. $\mathrm{Br} \mathrm{J}$ Ophthalmol 1983; 67: 37-42.

6 Brown GC, Felton SM, Benson WE. Reversible night blindness associated with intestinal bypass surgery. Am J Ophthalmol 1980; 89: 776-779.

7 Walt RP, Kemp CM, Lyness L, Bird AC, Sherlock S. Vitamin A treatment for night blindness in primary biliary cirrhosis. BMJ 1984; 288: 1030-1031.

8 Wachtmeister L, Bjorkhem I, Diczfalusy U, Emami A. Attempts to define the minimal serum level of vitamin A required for normal visual function in a patient with severe fat malabsorption. Acta Ophthalmol (Copenhagen) 1988; 66: 341-348.

9 Sandberg MA, Rosen JB, Berson EL. Cone and rod function in vitamin A deficiency with chronic alcoholism and in retinitis pigmentosa. Am J Ophthalmol 1977; 84: 658-665. 
10 Carr RE. Vitamin A deficiency. In: Heckinlively JR Arden GB (eds). Principles and Practice of Clinical Electrophysiology of Vision. Mosby Year Book: St Louis, MO, 1991 pp 737-740.

11 Newman NJ, Capone A, Leeper HF, O'Day DG, Mandell B, Lambert SR et al. Clinical and subclinical ophthalmic findings with retinol deficiency. Opthalmology 1994; 101: 1077-1083.

12 Spits Y, De Laey J-J, Leroy BP. Rapid recovery of night blindness due to obesity surgery after vitamin A repletion therapy. Br J Ophthamol 2004; 88: 583-585.

13 Marmor MF, Zrenner E. Standard for clinical electroretinography (1999 update). Doc Ophthalmol 1999; 97: 143-156.

14 Bach M, Hawlina M, Holder GE, Marmour M, Meigan T, Vaegan et al. Standard for pattern electroretinography. Doc Ophthalmol 2000; 101: 11-18.

15 Seiving PA. Photopic ON- and OFF-pathway abnormalities in retinal dystrophies. Trans Am Ophthalmol Soc 1993; 91: 701-773.
16 Arden GE, Wolf J, Beringer T, Hog CR, Tzekov R, Holder GE. S-cone ERGs elicited by a simple technique in normals and in tritanopes. Vis Res 1998; 39: 641-650.

17 Arden GB, Gûndûz K, Perry S. Colour vision testing with a computer graphics system. Clin Vis Sci 1988; 2: 303-320.

18 Wali N, Leguire LE. The photopic hill: a new phenomenon of the light adapted electroretinogram. Doc Ophthalmol 1992; 80: 335-342.

19 Ueno S, Konodo M, Niwa Y, Terasaki H, Miyake Y. Luminance dependence of neural components that underlies the primate photopic electroretinogram. Invest Ophtnalmol Vis Sci 2004; 45: 1033-1040.

20 Nakamura M, Skalet J, Miyake Y. RDH5 mutations and electroretinograms in fundus albipunctatus with or without macular dystrophy. Doc Ophthalmol 2003; 107: 3-11.

21 Mata N, Radu R, Clemmons R, Travis GH. Isomerization and oxidation of vitamin $\mathrm{A}$ in cone-dominant retinas. A novel pathway for visual-pigment regeneration in daylight. Neuron 2002; 36: 69-80. 